\title{
Racial and Ethnic Disparities in Maternal Outcomes and the Disadvantage of Peripartum Black Women: A Multistate Analysis, 2007-2014
}

\author{
Virginia Tangel, MA ${ }^{1}$ Robert S. White, MD, MS ${ }^{2}$ Anna S. Nachamie, BS ${ }^{1}$ Jeremy S. Pick, MD ${ }^{2}$ \\ ${ }^{1}$ Center for Perioperative Outcomes, Department of Anesthesiology, \\ Weill Cornell Medicine, New York, New York \\ 2 Department of Anesthesiology, NewYork-Presbyterian Hospital/ \\ Weill Cornell Medicine, New York, New York \\ Address for correspondence Robert S. White, MD, MS, Department of \\ Anesthesiology, NewYork-Presbyterian Hospital/Weill Cornell \\ Medicine, 525 East 68th Street, New York, NY 10065 \\ (e-mail: rsw33@cornell.edu).
}

Am J Perinatol 2019;36:835-848.

\author{
Abstract \\ Keywords \\ - racial/ethnic \\ disparities \\ - in-hospital maternal \\ mortality \\ - cesarean delivery \\ - administrative \\ database \\ - outcomes research \\ - maternal length of \\ stay
}

Objective Racial and ethnic disparities in obstetric care and delivery outcomes have shown that black women experience high rates of pregnancy-related mortality and morbidity, along with high rates of cesarean delivery, compared with other racial and ethnic groups. We aimed to quantify these disparities and test the effects of race/ ethnicity in stratified statistical models by insurance payer and socioeconomic status, adjusting for comorbidities specific to an obstetric population.

Study Design We analyzed maternal outcomes in a sample of 6,872,588 delivery records from California, Florida, Kentucky, Maryland, and New York from 2007 to 2014 from the State Inpatient Databases, Healthcare Cost and Utilization Project. We compared present-on-admission characteristics of parturients by race/ethnicity, and estimated logistic regression and generalized linear models to assess outcomes of inhospital mortality, cesarean delivery, and length of stay.

Results Compared with white women, black women were more likely to die inhospital (odds ratio [OR]: 1.90, 95\% confidence interval [Cl]: 1.47-2.45) and have a longer average length of stay (incidence rate ratio: $1.10,95 \% \mathrm{Cl}: 1.09-1.10)$. Black women also were more likely to have a cesarean delivery (OR: $1.12,95 \% \mathrm{Cl} 1.12-1.13$ ) than white women. These results largely held in stratified analyses.

Conclusion In most insurance payers and socioeconomic strata, race/ethnicity alone is a factor that predicts parturient outcomes.
Racial and ethnic disparities in obstetric care and delivery outcomes have been previously reported, showing black women experience disproportionately higher rates of mortality and morbidity associated with pregnancy than all other racial and ethnic groups. ${ }^{1-9}$ In the United States, black women are two to four times more likely than white women to die in the peripartum period due to cardiovascular, cerebrovascular, and pulmonary complications. ${ }^{1,3,10-15}$

In addition, studies have identified black parturients to be more likely than women from other racial and ethnic groups to have a cesarean delivery, even when controlling for

received

June 6, 2018

accepted after revision

September 13, 2018

published online

January 14, 2019 comorbidities. ${ }^{1,16-25}$ Cesarean delivery is more deleterious to maternal health than vaginal deliveries due to an increased risk of poor outcomes with subsequent pregnancies, longer recovery times, and longer hospital lengths of stay (LOS). ${ }^{26,27}$ Black women are also more likely to be readmitted after cesarean and vaginal delivery ${ }^{28}$ and have longer postpartum hospital LOS. ${ }^{7}$

The disproportionately poor maternal outcomes experienced by black women have been attributed to the cumulative effects of structural racism. ${ }^{29-36}$ Demographically, black women are more likely to live in socioeconomically
Copyright $\odot 2019$ by Thieme Medical Publishers, Inc., 333 Seventh Avenue, New York, NY 10001, USA. Tel: +1(212) 584-4662.
DOI https://doi.org/ 10.1055/s-0038-1675207. ISSN 0735-1631. 
disadvantaged areas ${ }^{37}$ and are less likely to have private insurance as their primary insurance payer than white women. ${ }^{38}$ These factors result in poorer access to quality of care than other racial and ethnic groups. ${ }^{39}$ Additionally, providers' internalized views of their patients' racial or ethnic identity potentially result in implicit bias leading to a further layer of substandard care. ${ }^{33,35,40}$ These forces interact in a lived experience of race and ethnicity that affects obstetric care and influences maternal health outcomes. $^{41}$

Although previous studies have controlled for income and insurance payer as confounders of maternal outcomes, none have examined them as effect measure modifiers of race and ethnicity. ${ }^{7,21}$ Research utilizing data from large, representative data sets is needed to determine if these differences in outcomes by race and ethnicity, along with the disadvantage experienced by black women, are due to the unique experience of race and ethnicity alone, or if the effects of race and ethnicity are modified by primary insurance type and/or socioeconomic status. ${ }^{42,43}$

Using administrative discharge records of more than 6.8 million singleton and multiple deliveries in five states in the United States over an 8-year period, we aimed to expand upon existing literature by quantifying differences in maternal outcomes between racial and ethnic groups by in-hospital mortality, delivery mode, and hospital LOS. To accomplish this task, we used data from the State Inpatient Database (SID), Healthcare Cost and Utilization Project (HCUP), and Agency for Healthcare Quality and Research (AHRQ); a multistate database that approximates a census of inpatient hospital discharges in participating states. ${ }^{44}$ We hypothesized that racial and ethnic differences exist in outcomes in a population of peripartum patients, and that black patients experience poorer outcomes as compared with white patients. In particular, we hypothesized that these differences result in increased rates of in-hospital mortality, cesarean deliveries, and longer LOS, despite controlling for potential confounders such as present-on-admission comorbidities or when assessing effect modification (through stratified statistical models) by insurance payer and socioeconomic status. Our efforts update numbers on outcomes in a peripartum population, as well as assess the homogeneity of the effects of race/ethnicity on these outcomes by levels of insurance payer and socioeconomic status.

\section{Materials and Methods}

We conducted a retrospective analysis of inpatient singleton and multiple deliveries from patients aged $\geq 18$ years (International Classification of Diseases, Ninth Revision, Clinical Modification [ICD-9-CM]) using data from the SIDs (SID, HCUP, AHRQ) from the years 2007 to 2011 for California, and years 2007 to 2014 for Florida, New York, Maryland, and Kentucky. ${ }^{44}$ The SID is an administrative database that contains information on comorbidities, diagnoses, procedures, and complications of procedures (all of which are identified by ICD-9-CM codes); an indicator to distinguish present-on-admission comorbidities and diagnoses, hospital characteristics; patient demographics; dates of admission and discharge; hospital LOS; and disposition at discharge. ${ }^{44}$ Records are by hospital admission.

Singleton deliveries were identified by ICD-9-CM codes V27.0 and V27.1, and multiple births were identified by ICD9-CM codes V27.2-8. Deliveries were categorized into cesarean (ICD-9-CM 74.0-74.2, 74.4, 74.99), operative vaginal (ICD-9CM 72.0-72.9, 73.3), or normal vaginal deliveries. Ectopic and molar pregnancies, as well as terminated pregnancies, were excluded from analysis (ICD-9-CM 630-639, 69.01, 75.0).

The Institutional Review Board at Weill Cornell Medicine determined this research to be exempt from review due to its retrospective nature and lack of patient identifiers.

\section{Outcome Measures}

The primary outcome of our study was maternal inpatient mortality. Secondary outcomes included cesarean delivery versus a vaginal or operative vaginal delivery (i.e., forceps or vacuum-assisted) and hospital LOS.

\section{Patient and Hospital Variables}

The primary variable of interest was race/ethnicity, which was coded as white, black (Black or African American), Hispanic, and Other (including Asian, Native Hawaiian or Other Pacific Islander, Native American, and more than one race/ethnicity). Other patient demographics examined included parturient age; primary payer status (including Medicare, Medicaid, private insurance, uninsured [including self-pay and no charge], and Other [including Worker's Compensation, CHAMPUS, CHAMP-VA, Title V, and other government programs]); delivery type (vaginal, operative vaginal [including forceps and vacuum deliveries], cesarean); socioeconomic status (operationalized as the median household income quartile for the patient's ZIP code); hospital state; hospital delivery volume quartile; and year of delivery.

Present-on-admission comorbidities included the index developed by Bateman et al, ${ }^{45}$ a validated comorbidity score for use in obstetric populations. ${ }^{46}$ The Bateman index includes weighted measures that predict maternal end-organ injury or death, including severe preeclampsia or eclampsia, chronic congestive heart failure, congenital heart disease, pulmonary hypertension, chronic ischemic heart disease, sickle cell disease, multiple gestation, cardiac valvular disease, systemic lupus erythematosus, human immunodeficiency virus, mild or unspecified preeclampsia (without severe preeclampsia or eclampsia), drug abuse, placenta previa, chronic renal disease, preexisting hypertension, previous cesarean delivery, gestational hypertension, alcohol abuse, asthma, preexisting diabetes mellitus, and maternal age older than 35 (categorized as ages $35-39,40-44$, and $45+$ ).

\section{Statistical Analyses}

Descriptive statistics by racial/ethnic group and delivery type were calculated separately. Variables were compared in unadjusted analyses using the appropriate parametric and nonparametric tests for categorical and ordinal or continuous variables: Pearson's chi-square test, Fisher's exact test, one-way analysis of variance, Mann-Whitney $U$-test, or Kruskal-Wallis test. 


\section{Multivariate Models}

We fit multivariate logistic regression models to predict the outcomes of in-hospital mortality and cesarean deliveries. We fit multivariate generalized linear models with a log link that followed a negative binomial distribution to predict the count outcome of LOS.

Predictor variables in all multivariate models were chosen a priori; all were significantly different between racial/ethnic groups at $\alpha \leq 0.05$ : race (reference: white), primary payer (reference: private insurance), median household income quartile (reference: first quartile), patient age as a linear term, the maternal comorbidity index as defined by Bateman, ${ }^{45}$ hospital state (reference: Florida), delivery year (reference: 2007), and hospital delivery volume (reference: first quartile).

For each multivariate model, only observations with nonmissing covariate data were included. For each outcome, a model was run containing the aforementioned predictor variables. Each model contained only these predictor variables; a variable indicating delivery type (reference: vaginal delivery) was additionally included in models for the outcomes of inpatient mortality and LOS.

Subsequent stratified multivariate models for each outcome were run by subpopulations of social determinants of health: primary insurance payer and quartile of median income for the patient's residential ZIP code. These models contained all of the variables present in the main models, save for the variable by which they were stratified. Stratified models were used to assess potential heterogeneity in the effects of race/ethnicity between the main models and within each category of insurance payer and median income quartile.

For dichotomous outcomes, the area under the receiver operating characteristic curve was calculated to assess model discrimination. Assumptions regarding the distribution of the LOS were assessed graphically.

Data are expressed as $n$ (percent), mean (standard deviation), median (interquartile range [IQR]) in bivariate analyses; odds ratio (OR, 95\% confidence interval, $\mathrm{CI}$ ) for logistic regression models; or incidence rate ratio (IRR, 95\% CI) for generalized linear models with negative binomial distributions. All $p$-values are two-sided, and statistical significance is evaluated at the $0.05 \alpha$ level. Data analysis was conducted in SAS 9.4 (SAS Institute, Cary, NC) and in Stata SE 15 (StataCorp, College Station, TX).

\section{Results}

\section{Sample Attrition}

There were 7,109,892 records in the database with an identified singleton or multiple birth. After dropping cases with missing values on age, the sample size decreased to $7,097,656$. Following the exclusion criteria of Cheesman et al, we omitted cases of ectopic and molar pregnancies, abortions, dilation and curettage for termination of pregnancy, aspiration curettage for termination of pregnancy, hysterectomy to terminate pregnancy, and intra-amniotic injections for abortions, which reduced the sample size to
$7,097,179 .{ }^{47}$ The sample was further reduced to include only patients 18 years or older $(6,893,416)$, with a nonmissing value on gender $(6,875,717)$, a nonmissing final disposition $(6,875,593)$, nonmissing data on primary insurance payer $(6,874,806)$, and with no more than one type of preeclampsia coded on the record (including no preeclampsia). This resulted in a final sample size of 6,872,588.

\section{Descriptive Statistics}

A total of 6,872,588 patients were included in the sample; 2,922,680 (42.5\%) were white, 1,930,270 (28.1\%) were Hispanic, 957,513 (13.9\%) were black, and 871,624 (12.7\%) were of another race/ethnicity (-Table 1). In addition, 190,501 (2.8\%) of the sample had missing values on race/ethnicity. Black women had the highest percentage of cesarean deliveries, at $38.3 \%$, followed by Hispanic women, white women, and women of other races/ethnicities.

Hispanic women had the highest proportion of vaginal deliveries (excluding forceps or vacuum deliveries), at $60.8 \%$, followed by white women, women of other races/ ethnicities, and black women. Black women were most likely to live in the lowest quartile of median income, and women of other races/ethnicities were most likely to live in the top quartile of median income. Two-thirds of Hispanic women were insured by Medicaid, along with nearly the same percentage of black women. Approximately onethird of women of other races/ethnicities and white women were insured by Medicaid. White women had the highest proportion of all racial/ethnic groups of private insurance.

Each racial/ethnic group had the same median scores on the Bateman comorbidity index, which ranged in values from 0 to 21 (data not shown).

The largest proportion of cesarean deliveries in a population of black parturients were among those with Medicare or private insurance ( 45.2 and $42.6 \%$, respectively, unpublished data), compared with black women insured by Medicaid (36.4\%), with other types of insurance $(37.8 \%)$, or uninsured (33.6\%). By comparison, the largest proportion of cesarean deliveries in a population of white patients was with patients insured by Medicare (45.1\%), followed by private insurance (36.4\%), other insurance (32.2\%), Medicaid (32.1\%), and uninsured $(29.6 \%)$. In a Hispanic patient population, the highest proportion of those having a cesarean delivery were also on Medicare (46.5\%), followed by private insurance (38.2\%), other insurance (34.6\%), Medicaid (34.1\%), and uninsured (32.1\%). Finally, in a patient population of those from other races, $42.7 \%$ of Medicaid patients had cesarean delivery, followed by $35.5 \%$ of patients with private insurance, $31.8 \%$ with Medicaid, $34.1 \%$ with other types of insurance, and $30.9 \%$ of uninsured.

The rate of cesarean deliveries increased with each successive increase in median income quartile in a black patient population: $37.3 \%$ in the first quartile, $39.2 \%$ in the second quartile, $40.1 \%$ in the third quartile, and $41.2 \%$ in the fourth quartile. For white patients, the rate of cesarean deliveries followed approximately the same pattern: $32.8 \%$ in the first quartile, $34.2 \%$ in the second, $34.8 \%$ in the third, and $37.2 \%$ in 


\begin{tabular}{|c|c|c|c|c|c|c|c|c|c|c|c|c|c|c|c|c|c|c|c|c|c|c|c|c|c|c|c|}
\hline 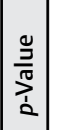 & & $\begin{array}{l}\bar{\delta} \\
\text { o. } \\
\dot{0} \\
v\end{array}$ & $\begin{array}{l}\bar{\delta} \\
\dot{0} \\
\dot{0} \\
\vee\end{array}$ & & & & $\begin{array}{l}\bar{\delta} \\
\varnothing \\
\dot{0} \\
\dot{v}\end{array}$ & & & & & & & & $\begin{array}{l}\bar{b} \\
\bar{o} \\
\dot{0} \\
v\end{array}$ & & & & & $\begin{array}{l}\bar{\delta} \\
\dot{0} \\
\dot{0} \\
v\end{array}$ & & & & $\begin{array}{l}\bar{\delta} \\
0 \\
0 \\
0 \\
v\end{array}$ & & & \\
\hline $\begin{array}{c} \\
\overline{\widetilde{m}} \\
\stackrel{0}{\circ}\end{array}$ & $\begin{array}{l}\widehat{O} \\
\dot{0} \\
0 \\
\stackrel{0}{0} \\
\infty \\
0 \\
0 \\
\mathbb{N} \\
\infty \\
0 \\
0\end{array}$ & 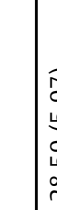 & & 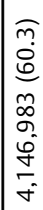 & 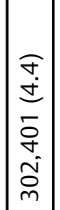 & 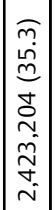 & $\begin{array}{ll}0 \\
0 \\
\vdots\end{array}$ & 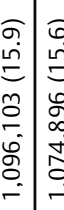 & 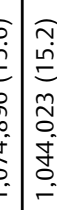 & 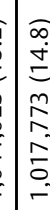 & 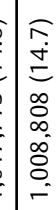 & 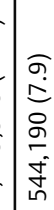 & 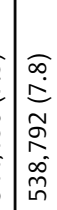 & 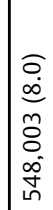 & & 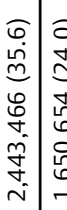 & 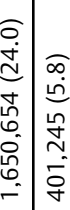 & 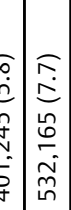 & 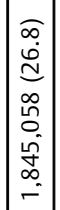 & 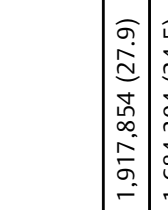 & 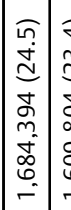 & 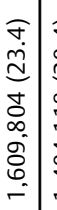 & 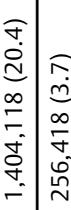 & & & 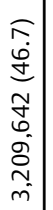 & 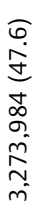 \\
\hline
\end{tabular}

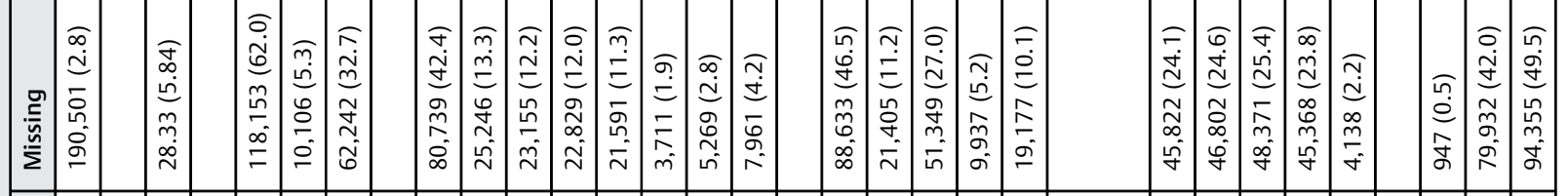

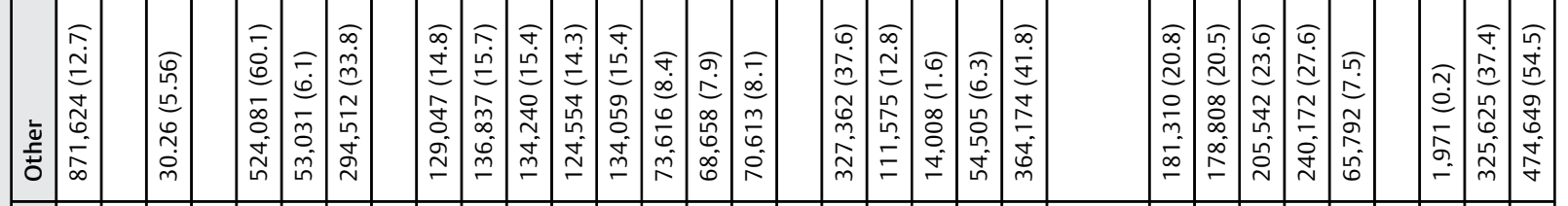

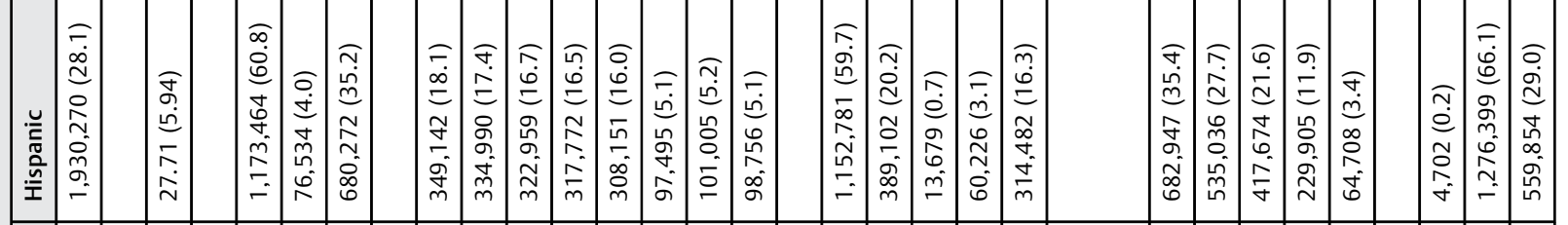

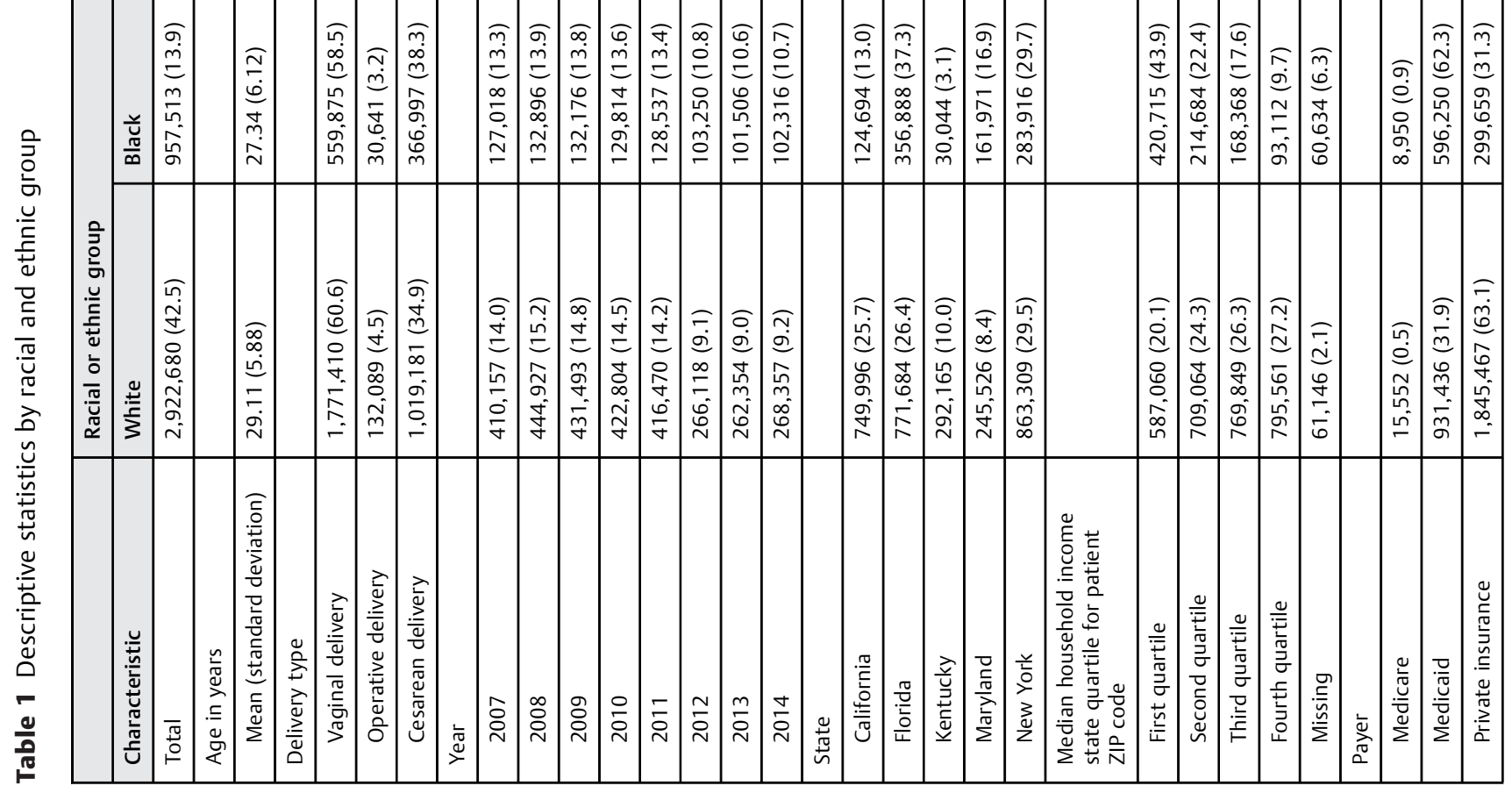




喜

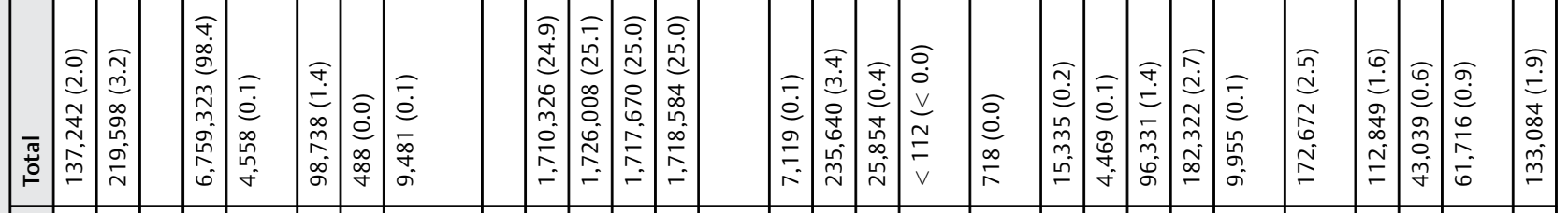

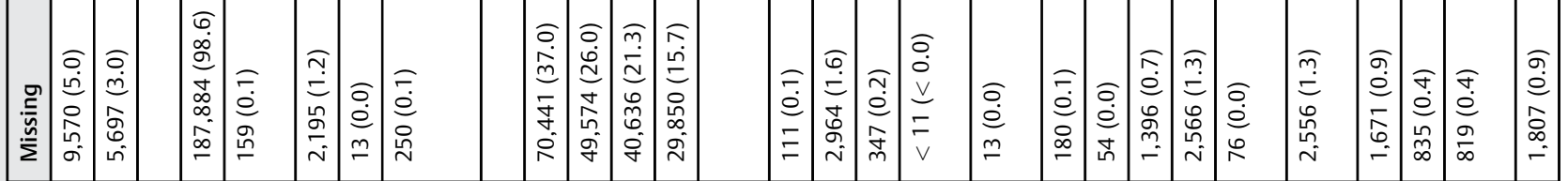

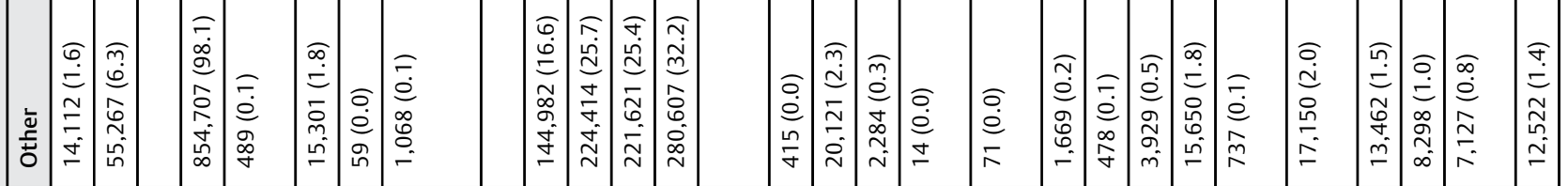

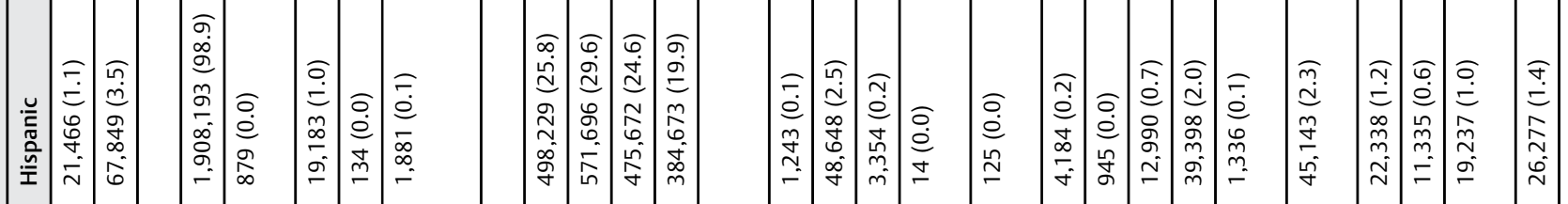

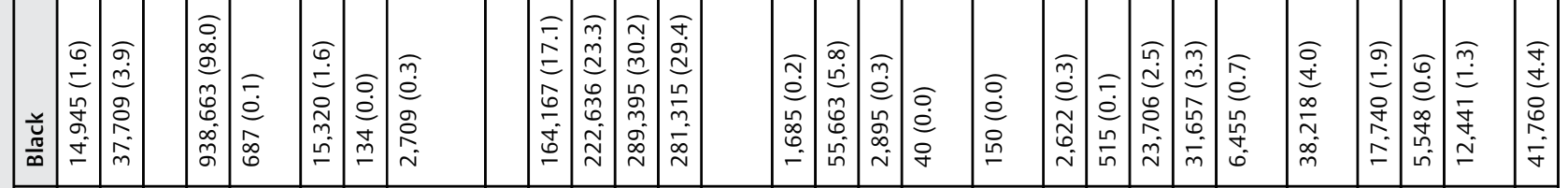

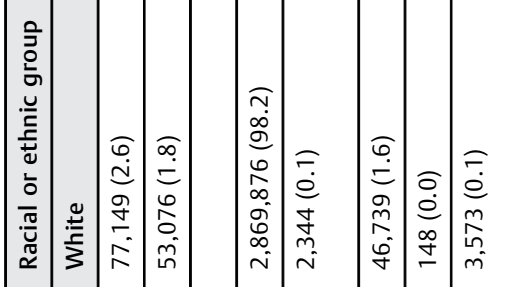

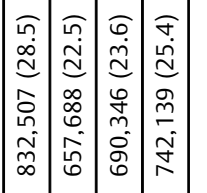




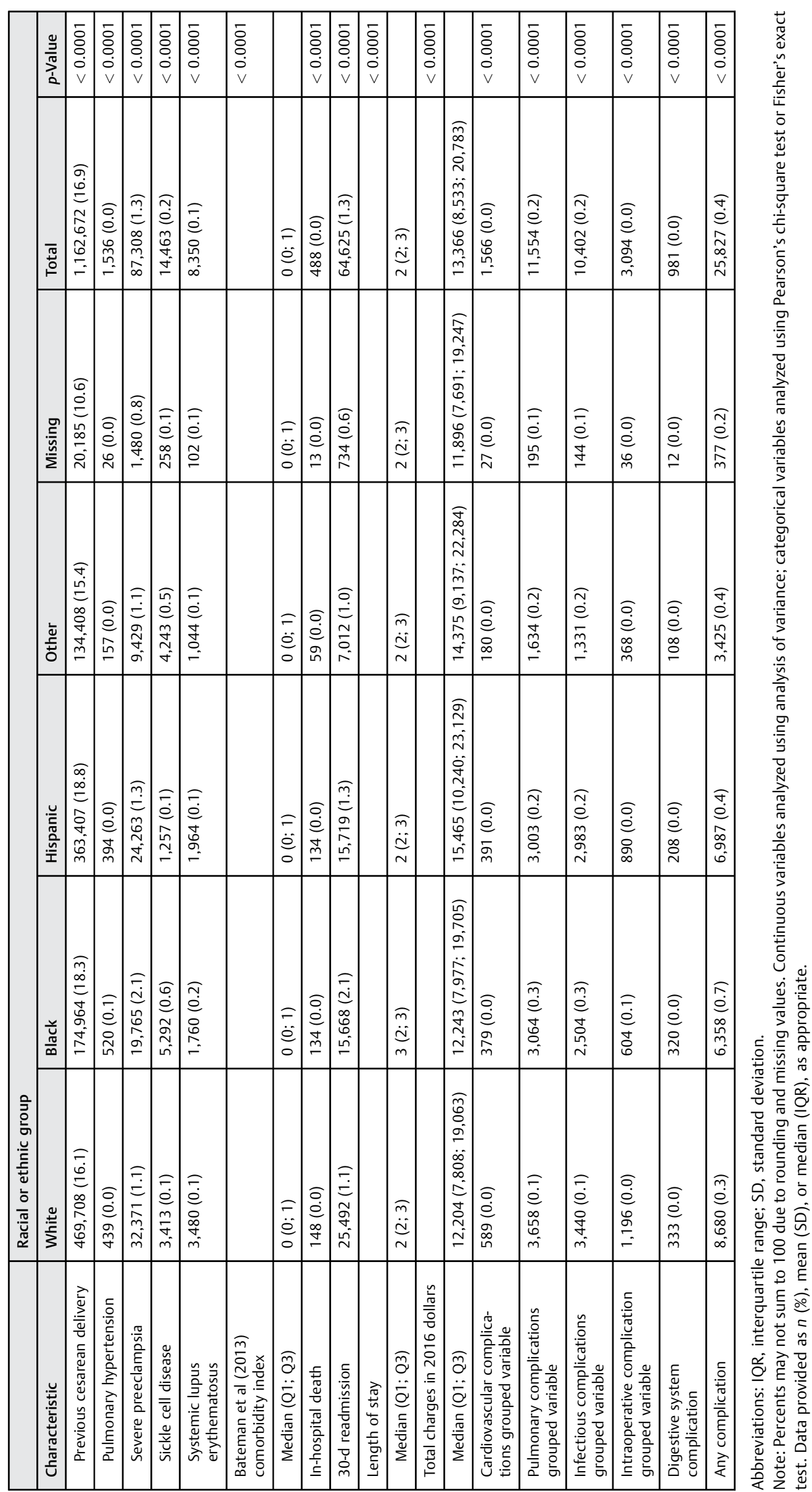


Table 2 Descriptive statistics by delivery type

\begin{tabular}{|c|c|c|c|c|c|}
\hline \multirow[b]{2}{*}{ Characteristic } & \multicolumn{5}{|l|}{ Delivery type } \\
\hline & Vaginal delivery & Operative vaginal & Cesarean delivery & Total & $p$-Value \\
\hline Total & $4,146,983(60.3)$ & $302,401(4.4)$ & $2,423,204(35.3)$ & $6,872,588(100.0)$ & \\
\hline Age in years & & & & & $<0.0001$ \\
\hline Mean (standard deviation) & $28.02(5.81)$ & $27.96(5.98)$ & $29.66(6.07)$ & 28.59 (5.97) & \\
\hline Race & & & & & $<0.0001$ \\
\hline White & $1,771,410(42.7)$ & $132,089(43.7)$ & $1,019,181(42.1)$ & $2,922,680(42.5)$ & \\
\hline Black & $559,875(13.5)$ & $30,641(10.1)$ & $366,997(15.1)$ & 957,513 (13.9) & \\
\hline Hispanic & $1,173,464(28.3)$ & $76,534(25.3)$ & $680,272(28.1)$ & $1,930,270(28.1)$ & \\
\hline Other & $524,081(12.6)$ & $53,031(17.5)$ & $294,512(12.2)$ & $871,624(12.7)$ & \\
\hline Missing & $118,153(2.8)$ & $10,106(3.3)$ & $62,242(2.6)$ & $190,501(2.8)$ & \\
\hline Year & & & & & $<0.0001$ \\
\hline 2007 & $665,124(16.0)$ & 55,559 (18.4) & $375,420(15.5)$ & 1,096,103 (15.9) & \\
\hline 2008 & 651,081 (15.7) & $51,370(17.0)$ & $372,445(15.4)$ & $1,074,896(15.6)$ & \\
\hline 2009 & $627,856(15.1)$ & 47,569 (15.7) & $368,598(15.2)$ & $1,044,023(15.2)$ & \\
\hline 2010 & $614,343(14.8)$ & $45,596(15.1)$ & $357,834(14.8)$ & $1,017,773(14.8)$ & \\
\hline 2011 & 609,179 (14.7) & 44,496 (14.7) & $355,133(14.7)$ & $1,008,808(14.7)$ & \\
\hline 2012 & $325,432(7.8)$ & $20,088(6.6)$ & $198,670(8.2)$ & 544,190 (7.9) & \\
\hline 2013 & $322,713(7.8)$ & $18,978(6.3)$ & $197,101(8.1)$ & $538,792(7.8)$ & \\
\hline 2014 & $331,255(8.0)$ & $18,745(6.2)$ & $198,003(8.2)$ & $548,003(8.0)$ & \\
\hline State & & & & & $<0.0001$ \\
\hline California & $1,492,973(36.0)$ & $135,345(44.8)$ & $815,148(33.6)$ & $2,443,466(35.6)$ & \\
\hline Florida & $949,901(22.9)$ & $56,154(18.6)$ & $644,599(26.6)$ & $1,650,654(24.0)$ & \\
\hline New York & $1,148,213(27.7)$ & $65,117(21.5)$ & $631,728(26.1)$ & $1,845,058(26.8)$ & \\
\hline $\begin{array}{l}\text { Median household income state quartile for patient } \\
\text { ZIP code }\end{array}$ & & & & & $<0.0001$ \\
\hline First quartile & $1,178,675(28.4)$ & $77,658(25.7)$ & $661,521(27.3)$ & $1,917,854$ (27.9) & \\
\hline Second quartile & $1,018,750(24.6)$ & $76,674(25.4)$ & $588,970(24.3)$ & $1,684,394(24.5)$ & \\
\hline Third quartile & $966,701(23.3)$ & $73,424(24.3)$ & $569,679(23.5)$ & $1,609,804(23.4)$ & \\
\hline Fourth quartile & $817,882(19.7)$ & $65,452(21.6)$ & $520,784(21.5)$ & $1,404,118(20.4)$ & \\
\hline Missing & $164,975(4.0)$ & $9,193(3.0)$ & $82,250(3.4)$ & $256,418(3.7)$ & \\
\hline Payer & & & & & $<0.0001$ \\
\hline Medicare & $16,489(0.4)$ & $1,150(0.4)$ & $14,483(0.6)$ & $32,122(0.5)$ & \\
\hline Medicaid & $2,000,245(48.2)$ & $129,712(42.9)$ & $1,079,685(44.6)$ & $3,209,642(46.7)$ & \\
\hline Private insurance & $1,903,782(45.9)$ & $155,997(51.6)$ & $1,214,205(50.1)$ & $3,273,984(47.6)$ & \\
\hline Other & $84,793(2.0)$ & $6,403(2.1)$ & $46,046(1.9)$ & $137,242(2.0)$ & \\
\hline Self-pay/No charge & $141,674(3.4)$ & $9,139(3.0)$ & $68,785(2.8)$ & $219,598(3.2)$ & \\
\hline Disposition at discharge & & & & & $<0.0001$ \\
\hline Routine & $4,083,569(98.5)$ & 297,727 (98.5) & $2,378,027(98.1)$ & $6,759,323(98.4)$ & \\
\hline Transfer to short-term hospital & $1,483(0.0)$ & $149(0.0)$ & $2,926(0.1)$ & $4,558(0.1)$ & \\
\hline Home health care & $56,371(1.4)$ & $4,200(1.4)$ & $38,167(1.6)$ & $98,738(1.4)$ & \\
\hline Died & $110(0.0)$ & $12(0.0)$ & $366(0.0)$ & $488(0.0)$ & \\
\hline $\begin{array}{l}\text { Transfer to other, against medical advice, } \\
\text { or destination unknown }\end{array}$ & $5,450(0.1)$ & $313(0.1)$ & $3,718(0.2)$ & $9,481(0.1)$ & \\
\hline Hospital volume & & & & & $<0.0001$ \\
\hline
\end{tabular}


842 Racial and Ethnic Disparities in Maternal Outcomes Tangel et al.

Table 2 (Continued)

\begin{tabular}{|c|c|c|c|c|c|}
\hline \multirow[b]{2}{*}{ Characteristic } & \multicolumn{5}{|l|}{ Delivery type } \\
\hline & Vaginal delivery & Operative vaginal & Cesarean delivery & Total & $p$-Value \\
\hline Third quartile & $1,048,627(25.3)$ & $68,085(22.5)$ & $600,958(24.8)$ & $1,717,670(25.0)$ & \\
\hline Fourth quartile & $1,016,021(24.5)$ & $70,286(23.2)$ & $632,277(26.1)$ & $1,718,584(25.0)$ & \\
\hline \multicolumn{6}{|l|}{ Bateman et al (2013) comorbidities } \\
\hline Alcohol abuse & $4,384(0.1)$ & $226(0.1)$ & $2,509(0.1)$ & $7,119(0.1)$ & $<0.0001$ \\
\hline Asthma & $132,142(3.2)$ & $7,927(2.6)$ & $95,571(3.9)$ & $235,640(3.4)$ & $<0.0001$ \\
\hline Cardiac valvular disease & $13,627(0.3)$ & $1,120(0.4)$ & $11,107(0.5)$ & $25,854(0.4)$ & $<0.0001$ \\
\hline Chronic congestive heart failure & $24(0.0)$ & $<11(<0.0)$ & $72(0.0)$ & $<107(<0.0)$ & $<0.0001$ \\
\hline Chronic ischemic heart disease & $260(0.0)$ & $39(0.0)$ & $419(0.0)$ & $718(0.0)$ & $<0.0001$ \\
\hline Chronic renal disease & $7,727(0.2)$ & $552(0.2)$ & $7,056(0.3)$ & $15,335(0.2)$ & $<0.0001$ \\
\hline Congenital heart disease & $2,192(0.1)$ & $302(0.1)$ & $1,975(0.1)$ & $4,469(0.1)$ & $<0.0001$ \\
\hline Drug abuse & $58,905(1.4)$ & $3,352(1.1)$ & $34,074(1.4)$ & $96,331(1.4)$ & $<0.0001$ \\
\hline Gestational hypertension & $97,410(2.3)$ & $8,126(2.7)$ & $76,786(3.2)$ & $182,322(2.7)$ & $<0.0001$ \\
\hline Human immunodeficiency virus & $3,845(0.1)$ & $96(0.0)$ & $6,014(0.2)$ & $9,955(0.1)$ & $<0.0001$ \\
\hline Mild or unspecified preeclampsia & $74,957(1.8)$ & $6,517(2.2)$ & $91,198(3.8)$ & $172,672(2.5)$ & $<0.0001$ \\
\hline Multiple gestation & $21,207(0.5)$ & $3,135(1.0)$ & $88,507(3.7)$ & $112,849(1.6)$ & $<0.0001$ \\
\hline Placenta previa & $5,829(0.1)$ & $399(0.1)$ & $36,811(1.5)$ & $43,039(0.6)$ & $<0.0001$ \\
\hline Preexisting diabetes mellitus & $20,426(0.5)$ & $1,557(0.5)$ & $39,733(1.6)$ & $61,716(0.9)$ & $<0.0001$ \\
\hline Preexisting hypertension & $53,498(1.3)$ & $3,319(1.1)$ & $76,267(3.1)$ & $133,084(1.9)$ & $<0.0001$ \\
\hline Previous cesarean delivery & $89,409(2.2)$ & $8,230(2.7)$ & $1,065,033(44.0)$ & $1,162,672(16.9)$ & $<0.0001$ \\
\hline Pulmonary hypertension & $475(0.0)$ & $100(0.0)$ & $961(0.0)$ & $1,536(0.0)$ & $<0.0001$ \\
\hline Severe preeclampsia & $24,111(0.6)$ & $2,151(0.7)$ & $61,046(2.5)$ & $87,308(1.3)$ & $<0.0001$ \\
\hline Sickle cell disease & $7,748(0.2)$ & $613(0.2)$ & $6,102(0.3)$ & $14,463(0.2)$ & $<0.0001$ \\
\hline Systemic lupus erythematosus & $3,978(0.1)$ & $271(0.1)$ & $4,101(0.2)$ & $8,350(0.1)$ & $<0.0001$ \\
\hline Bateman et al (2013) comorbidity index & & & & & $<0.0001$ \\
\hline Median (Q1; Q3) & $0(0 ; 1)$ & $0(0 ; 1)$ & $1(0 ; 2)$ & $0(0 ; 1)$ & \\
\hline In-hospital death & $110(0.0)$ & $12(0.0)$ & $366(0.0)$ & $488(0.0)$ & $<0.0001$ \\
\hline 30-d readmission & $27,486(0.9)$ & $2,308(1.1)$ & $34,831(1.9)$ & $64,625(1.3)$ & $<0.0001$ \\
\hline Length of stay & & & & & $<0.0001$ \\
\hline Median (Q1; Q3) & $2(2 ; 3)$ & $2(2 ; 3)$ & $3(3 ; 4)$ & $2(2 ; 3)$ & \\
\hline Total charges in 2016 dollars & & & & & $<0.0001$ \\
\hline Median (Q1; Q3) & $\begin{array}{l}10,986 \\
(7,414 ; 16,100)\end{array}$ & $\begin{array}{l}12,693 \\
(8,308 ; 18,675)\end{array}$ & $\begin{array}{l}19,788 \\
(12,765 ; 28,280)\end{array}$ & $\begin{array}{l}13,366 \\
(8,533 ; 20,783)\end{array}$ & \\
\hline Cardiovascular complications grouped variable & $451(0.0)$ & $52(0.0)$ & $1,063(0.0)$ & $1,566(0.0)$ & $<0.0001$ \\
\hline Pulmonary complications grouped variable & $1,547(0.0)$ & $204(0.1)$ & $9,803(0.4)$ & $11,554(0.2)$ & $<0.0001$ \\
\hline Infectious complications grouped variable & $3,574(0.1)$ & $327(0.1)$ & $6,501(0.3)$ & $10,402(0.2)$ & $<0.0001$ \\
\hline Intraoperative complication grouped variable & $306(0.0)$ & $28(0.0)$ & $2,760(0.1)$ & $3,094(0.0)$ & $<0.0001$ \\
\hline Digestive system complication & $53(0.0)$ & $<11(<0.0)$ & $921(0.0)$ & $<985(<0.0)$ & $<0.0001$ \\
\hline Any complication & $5,653(0.1)$ & $568(0.2)$ & $19,606(0.8)$ & $25,827(0.4)$ & $<0.0001$ \\
\hline
\end{tabular}

Abbreviations: IQR, interquartile range; SD, standard deviation.

Note: Percents may not sum to 100 due to rounding and missing values. Continuous variables analyzed using analysis of variance; categorical variables analyzed using Pearson's chi-square test or Fisher's exact test. Data provided as n (\%), mean (SD), or median (IQR), as appropriate.

the fourth. Among Hispanic patients, $35.2 \%$ of the first income quartile, $35.0 \%$ in the second, $35.2 \%$ in the third, and $37.6 \%$ in the fourth had cesarean deliveries. Finally, those in other races/ethnicities had the lowest rates of cesarean deliveries across the board: $31.6 \%$ of the first quartile of median income, $34.4 \%$ of the second, $34.7 \%$ of the third, and $35.1 \%$ of the fourth.

Patients with vaginal or operative deliveries had a median score of 0 (IQR: $0 ; 1)$ on the Bateman comorbidity index, while cesarean deliveries had a 1 (IQR: 0; 2) (-Table 2). 
Less than $1 \%$ of the women in the entire sample died inhospital (-Table 1). In an overall sample, black women had the longest LOS of all racial/ethnic groups.

\section{Multivariate Results}

\section{In-Hospital Mortality}

After adjustment for patient-level (race [reference: white], primary payer [reference: private insurance], median household income quartile [reference: first quartile], patient age as a linear term, the maternal comorbidity index as defined by Bateman, ${ }^{45}$ and delivery type [reference: vaginal]) and hospital-level variables (hospital state [reference: Florida], delivery year [reference: 2007], and hospital delivery volume [reference: first quartile]), black patients were 90\% more likely to die in-hospital when compared with white women (-Table 3). Each incremental increase on the Bateman comorbidity index resulted in a $45 \%$ increase in the likelihood of in-hospital maternal mortality (OR: 1.45, 95\% CI: $1.39-1.51, p<0.001$, data not shown). Stratified subgroup analysis by insurance status showed that black women with Medicaid insurance and black women with private insurance had increased odds of in-hospital death when compared with white women with those respective insurances. In a sample of Medicaid patients only, black women were $61 \%$ more likely than white women to die in-hospital; black women and women of other races/ethnicities (individually) were more than $100 \%$ more likely than white women to die in-hospital in a sample of patients with private insurance. In a population of patients with other insurance, Hispanic women were more than four times as likely as white women to die in-hospital.

In populations of the lowest and highest income quartiles, respectively, no racial/ethnic group was significantly more or less likely to die in-hospital as compared with white patients, though this negative finding could be attributed to the small number of individuals in the entire sample who died in the inpatient setting.

\section{Delivery Type}

After adjustment for patient-level (race [reference: white], primary payer [reference: private insurance], median household income quartile [reference: first quartile], patient age as a linear term, the maternal comorbidity index as defined by Bateman ${ }^{45}$ ) and hospital-level variables (hospital state [reference: Florida], delivery year [reference: 2007], and hospital delivery volume [reference: first quartile]), $6,872,588$ women had nonmissing data on all model covariates and were included in our analysis. Black and Hispanic women were 12 and $23 \%$ more likely, respectively, when compared with white women to have undergone a cesarean delivery as compared with a vaginal delivery (- Table 4). Women of other races/ethnicities were also more likely than white women to have an increased odds of cesarean delivery. For each incremental increase on the Bateman comorbidity index, women became 2.20 times more likely to have a cesarean delivery (OR: 2.20, 95\% CI: 2.20-2.20, $p<0.001$, data not shown).

Table 3 Risk-adjusted outcomes of in-hospital mortality according to race and ethnicity

\begin{tabular}{|l|l|l|l|l|l|l|}
\hline Population & White (reference) & Black & Hispanic & Other & Missing & $N$ \\
\hline Full sample & 1.00 & $\begin{array}{l}1.90^{\mathrm{c}} \\
(1.47,2.45)\end{array}$ & $\begin{array}{l}1.17 \\
(0.91,1.52)\end{array}$ & $\begin{array}{l}1.33 \\
(0.97,1.82)\end{array}$ & $\begin{array}{l}1.44 \\
(0.81,2.56)\end{array}$ & $6,872,588$ \\
\hline Medicare only & 1.00 & $\begin{array}{l}0.47 \\
(0.08,2.62)\end{array}$ & $\begin{array}{l}0.56 \\
(0.06,5.12)\end{array}$ & $\begin{array}{l}1.00 \\
(1.00,1.00)\end{array}$ & $\begin{array}{l}1.00 \\
(1.00,1.00)\end{array}$ & 9,823 \\
\hline Medicaid only & 1.00 & $\begin{array}{l}1.61^{\mathrm{b}} \\
(1.15,2.27)\end{array}$ & $\begin{array}{l}0.91 \\
(0.64,1.30)\end{array}$ & $\begin{array}{l}1.08 \\
(0.66,1.76)\end{array}$ & $\begin{array}{l}0.75 \\
(0.27,2.08)\end{array}$ & $3,209,642$ \\
\hline Private insurance only & 1.00 & $\begin{array}{l}2.52^{\mathrm{c}} \\
(1.65,3.84)\end{array}$ & $\begin{array}{l}1.49 \\
(0.95,2.34)\end{array}$ & $\begin{array}{l}2.03^{\mathrm{b}} \\
(1.31,3.15)\end{array}$ & $\begin{array}{l}3.05^{\mathrm{b}} \\
(1.45,6.43)\end{array}$ & $3,273,984$ \\
\hline Other insurance only & 1.00 & $\begin{array}{l}1.07 \\
(0.11,10.08)\end{array}$ & $\begin{array}{l}4.49^{\mathrm{a}} \\
(1.21,16.69)\end{array}$ & $\begin{array}{l}1.00 \\
(1.00,1.00)\end{array}$ & $\begin{array}{l}1.92 \\
(0.16,23.38)\end{array}$ \\
\hline Uninsured only & 1.00 & $\begin{array}{l}1.38 \\
(0.48,3.95)\end{array}$ & $\begin{array}{l}0.68 \\
(0.23,2.00)\end{array}$ & $\begin{array}{l}0.51 \\
(0.14,1.90)\end{array}$ & $\begin{array}{l}1.00 \\
(1.00,1.00)\end{array}$ & 213,901 \\
\hline $\begin{array}{l}\text { Median income } \\
\text { quartile 1 (lowest) only }\end{array}$ & 1.00 & $\begin{array}{l}1.45 \\
(0.97,2.16)\end{array}$ & $\begin{array}{l}0.93 \\
(0.61,1.43)\end{array}$ & $\begin{array}{l}0.81 \\
(0.41,1.59)\end{array}$ & $\begin{array}{l}0.84 \\
(0.26,2.73)\end{array}$ \\
\hline $\begin{array}{l}\text { Median income } \\
\text { quartile 2 only }\end{array}$ & 1.00 & $\begin{array}{l}1.84^{\mathrm{a}} \\
(1.02,3.33)\end{array}$ & $\begin{array}{l}1.77^{\mathrm{a}} \\
(1.04,3.01)\end{array}$ & $\begin{array}{l}2.72^{\mathrm{b}} \\
(1.48,4.98)\end{array}$ & $\begin{array}{l}1.09 \\
(0.25,4.65)\end{array}$ & $1,917,854$ \\
\hline $\begin{array}{l}\text { Median income } \\
\text { quartile 3 only }\end{array}$ & 1.00 & $\begin{array}{l}3.32^{\mathrm{c}} \\
(2.01,5.49)\end{array}$ & $\begin{array}{l}1.01 \\
(0.56,1.84)\end{array}$ & $\begin{array}{l}2.12^{\mathrm{a}} \\
(1.18,3.81)\end{array}$ & $\begin{array}{l}1.06 \\
(0.25,4.50)\end{array}$ \\
\hline $\begin{array}{l}\text { Median income } \\
\text { quartile 4 (highest) only }\end{array}$ & 1.00 & $\begin{array}{l}0.92 \\
(0.35,2.46)\end{array}$ & $\begin{array}{l}0.98 \\
(0.48,1.98)\end{array}$ & $\begin{array}{l}0.51 \\
(0.20,1.33)\end{array}$ & $\begin{array}{l}3.38^{\mathrm{a}} \\
(1.28,8.91)\end{array}$ \\
\hline
\end{tabular}

Abbreviation: $\mathrm{Cl}$, confidence interval.

Note: All numbers reported are odds ratios $(95 \% \mathrm{Cl})$ unless noted otherwise.

${ }^{\mathrm{a}} \mathrm{p}<0.05$.

${ }^{\mathrm{b}} \mathrm{p}<0.01$

${ }^{c} p<0.001$ 
Table 4 Risk-adjusted outcomes of having a cesarean delivery (vs. vaginal or operative vaginal delivery) according to race and ethnicity

\begin{tabular}{|c|c|c|c|c|c|c|}
\hline Population & White (reference) & Black & Hispanic & Other & Missing & $N$ \\
\hline Full sample & 1.00 & $\begin{array}{l}1.12^{\mathrm{c}} \\
(1.12,1.13)\end{array}$ & $\begin{array}{l}1.23^{\mathrm{c}} \\
(1.22,1.23)\end{array}$ & $\begin{array}{l}1.08^{c} \\
(1.07,1.08)\end{array}$ & $\begin{array}{l}1.11^{\mathrm{c}} \\
(1.09,1.12)\end{array}$ & $6,872,588$ \\
\hline Medicare only & 1.00 & $\begin{array}{l}0.88^{c} \\
(0.83,0.93)\end{array}$ & $\begin{array}{l}1.07 \\
(0.99,1.15)\end{array}$ & $\begin{array}{l}0.94 \\
(0.84,1.03)\end{array}$ & $\begin{array}{l}1.03 \\
(0.89,1.18)\end{array}$ & 32,122 \\
\hline Medicaid only & 1.00 & $\begin{array}{l}1.14^{\mathrm{c}} \\
(1.13,1.14)\end{array}$ & $\begin{array}{l}1.23^{\mathrm{c}} \\
(1.22,1.24)\end{array}$ & $\begin{array}{l}1.15^{\mathrm{c}} \\
(1.14,1.16)\end{array}$ & $\begin{array}{l}1.12^{\mathrm{C}} \\
(1.10,1.14)\end{array}$ & $3,209,642$ \\
\hline Private insurance only & 1.00 & $\begin{array}{l}1.16^{\mathrm{C}} \\
(1.15,1.17)\end{array}$ & $\begin{array}{l}1.20^{\mathrm{c}} \\
(1.19,1.21)\end{array}$ & $\begin{array}{l}1.05^{\mathrm{c}} \\
(1.04,1.06)\end{array}$ & $\begin{array}{l}1.09^{c} \\
(1.07,1.10)\end{array}$ & $3,273,984$ \\
\hline Other insurance only & 1.00 & $\begin{array}{l}1.15^{c} \\
(1.11,1.20)\end{array}$ & $\begin{array}{l}1.15^{c} \\
(1.11,1.19)\end{array}$ & $\begin{array}{l}1.06^{\mathrm{b}} \\
(1.02,1.10)\end{array}$ & $\begin{array}{l}1.16^{c} \\
(1.10,1.23)\end{array}$ & 137,242 \\
\hline Uninsured only & 1.00 & $\begin{array}{l}1.17^{\mathrm{c}} \\
(1.13,1.21)\end{array}$ & $\begin{array}{l}1.26^{\mathrm{c}} \\
(1.23,1.30)\end{array}$ & $\begin{array}{l}1.24^{\mathrm{c}} \\
(1.20,1.28)\end{array}$ & $\begin{array}{l}1.07^{\mathrm{a}} \\
(1.00,1.14)\end{array}$ & 219,598 \\
\hline $\begin{array}{l}\text { Median income } \\
\text { quartile } 1 \text { (lowest) only }\end{array}$ & 1.00 & $\begin{array}{l}1.15^{\mathrm{c}} \\
(1.14,1.16)\end{array}$ & $\begin{array}{l}1.28^{\mathrm{c}} \\
(1.27,1.29)\end{array}$ & $\begin{array}{l}1.11^{\mathrm{c}} \\
(1.10,1.13)\end{array}$ & $\begin{array}{l}1.11^{\mathrm{c}} \\
(1.09,1.14)\end{array}$ & $1,917,854$ \\
\hline $\begin{array}{l}\text { Median income } \\
\text { quartile } 2 \text { only }\end{array}$ & 1.00 & $\begin{array}{l}1.15^{c} \\
(1.14,1.16)\end{array}$ & $\begin{array}{l}1.18^{c} \\
(1.17,1.19)\end{array}$ & $\begin{array}{l}1.11^{\mathrm{c}} \\
(1.09,1.12)\end{array}$ & $\begin{array}{l}1.11^{\mathrm{c}} \\
(1.09,1.13)\end{array}$ & $1,684,394$ \\
\hline $\begin{array}{l}\text { Median income } \\
\text { quartile } 3 \text { only }\end{array}$ & 1.00 & $\begin{array}{l}1.20^{\mathrm{C}} \\
(1.18,1.21)\end{array}$ & $\begin{array}{l}1.23^{\mathrm{c}} \\
(1.22,1.24)\end{array}$ & $\begin{array}{l}1.10^{\mathrm{c}} \\
(1.09,1.12)\end{array}$ & $\begin{array}{l}1.11^{\mathrm{c}} \\
(1.08,1.13)\end{array}$ & $1,609,804$ \\
\hline $\begin{array}{l}\text { Median income } \\
\text { quartile } 4 \text { (highest) only }\end{array}$ & 1.00 & $\begin{array}{l}1.14^{\mathrm{c}} \\
(1.12,1.16)\end{array}$ & $\begin{array}{l}1.19^{c} \\
(1.18,1.20)\end{array}$ & $\begin{array}{l}1.04^{\mathrm{c}} \\
(1.03,1.05)\end{array}$ & $\begin{array}{l}1.13^{c} \\
(1.10,1.15)\end{array}$ & $1,404,118$ \\
\hline
\end{tabular}

Abbreviation: $\mathrm{Cl}$, confidence interval.

Note: All numbers reported are odds ratios $(95 \% \mathrm{Cl})$ unless noted otherwise.

${ }^{\mathrm{a}} p<0.05$.

${ }^{\mathrm{b}} p<0.01$.

${ }^{c} p<0.001$.

Black women, Hispanic women, and women of other races/ethnicities continued to have increased odds of cesarean delivery compared with white women when stratified subgroup analysis were performed by primary payer status for Medicaid insurance, private insurance, other insurance, and in an uninsured population. However, in a population of Medicare patients, black women had reduced odds of cesarean delivery compared with white women. Black women, Hispanic women, and women of other races/ethnicities were also individually more likely than white women to have increased odds of a cesarean delivery in each quartile of median income, with Hispanic women having the highest odds of a cesarean delivery as compared with white women in each quartile of median income.

\section{Length of Stay}

Models of LOS were interpreted using the IRR, which is the ratio of the average LOS for one group versus the average LOS for the reference group. In adjusted models (in which patientand hospital-level variables were controlled for-including delivery type), black women had an average LOS 1.10 times greater than white women ( - Table 5 ). This increase in average LOS was similarly significant in stratified analyses for each primary insurance payer type and quartile of median income. For each successively higher median income bracket as compared with the lowest bracket of median income, the average LOS for black as compared with white women decreased. For each successive increase in the Bateman comorbidity index, the average LOS increased by $8 \%$ (IRR: $1.08,95 \% \mathrm{CI}: 1.08-1.08$, $p<0.001$, data not shown).

Hispanic women and women of other races/ethnicities were expected to have slightly longer LOS than white women, though these findings were not uniform in stratified analyses by insurance payer and median income quartile.

\section{Discussion}

Using comprehensive discharge data from a geographically diverse population in the United States over an 8-year period, we identified disparities by race and ethnicity in maternal mortality, delivery type, and LOS.

For each of our outcomes, race/ethnicity served as an independent predictor when controlling for patient demographics, patient comorbidities specific to an obstetric population, and hospital-level variables. In stratified models, we analyzed outcomes in subgroups of insurance payer and median income quartile to assess the homogeneity of the effect of race/ethnicity across strata. Our findings suggest that race/ ethnicity is a significant predictor of in-hospital mortality, having a cesarean delivery, and having a longer expected LOS, even in many cases when testing for effect modification by insurance payer and median income category. Notably, our findings of the homogeneity of the effect of race/ethnicity in our primary outcome offer confirmatory evidence that black 
Table 5 Risk-adjusted outcomes of length of stay (count outcome) according to race and ethnicity

\begin{tabular}{|l|l|l|l|l|l|l|}
\hline Population & White (reference) & Black & Hispanic & Other & Missing & $N$ \\
\hline Full sample & 1.00 & $1.10^{c}$ & $1.01^{c}$ & $1.04^{c}$ & $1.01^{c}$ & $6,872,582$ \\
& & $(1.09,1.10)$ & $(1.01,1.01)$ & $(1.04,1.05)$ & $(1.01,1.02)$ & \\
\hline Medicare only & 1.00 & $1.10^{c}$ & 1.03 & 1.05 & 0.98 & 32,122 \\
& & $(1.07,1.14)$ & $(0.99,1.07)$ & $(1.00,1.11)$ & $(0.91,1.06)$ & \\
\hline Medicaid only & 1.00 & $1.10^{c}$ & $1.03^{c}$ & $1.05^{c}$ & $1.02^{c}$ & $3,209,640$ \\
& & $(1.09,1.10)$ & $(1.02,1.03)$ & $(1.04,1.05)$ & $(1.01,1.03)$ & \\
\hline Private insurance only & 1.00 & $1.10^{c}$ & $0.98^{c}$ & $1.05^{c}$ & 1.01 & $3,273,981$ \\
& & $(1.09,1.10)$ & $(0.98,0.98)$ & $(1.04,1.05)$ & $(1.00,1.02)$ & \\
\hline Other insurance only & 1.00 & $1.10^{c}$ & 1.02 & $1.03^{\mathrm{a}}$ & 1.00 & 137,242 \\
& & $(1.08,1.12)$ & $(1.00,1.04)$ & $(1.01,1.05)$ & $(0.97,1.03)$ & \\
\hline Uninsured only & 1.00 & $1.13^{c}$ & $1.06^{c}$ & $1.03^{c}$ & 1.03 & 219,597 \\
& & $(1.11,1.15)$ & $(1.04,1.07)$ & $(1.01,1.04)$ & $(0.99,1.06)$ & \\
\hline $\begin{array}{l}\text { Median income } \\
\text { quartile 1 (lowest) only }\end{array}$ & 1.00 & $1.11^{c}$ & $1.04^{c}$ & $1.05^{c}$ & $1.03^{c}$ & $1,917,854$ \\
\hline $\begin{array}{l}\text { Median income } \\
\text { quartile 2 only }\end{array}$ & 1.00 & $(1.10,1.12)$ & $(1.03,1.04)$ & $(1.04,1.06)$ & $(1.01,1.04)$ & \\
\hline $\begin{array}{l}\text { Median income } \\
\text { quartile 3 only }\end{array}$ & $1.09^{c}$ & $1.01^{\mathrm{b}}$ & $1.05^{\mathrm{c}}$ & $1.01^{\mathrm{a}}$ & $1,684,389$ \\
\hline $\begin{array}{l}\text { Median income } \\
\text { quartile 4 (highest) only }\end{array}$ & 1.00 & $(1.09,1.10)$ & $(1.00,1.01)$ & $(1.04,1.05)$ & $(1.00,1.03)$ & \\
\hline
\end{tabular}

Abbreviation: $\mathrm{Cl}$, confidence interval.

Note: All numbers reported are incidence rate ratios $(95 \% \mathrm{Cl})$ unless noted otherwise.

${ }^{a} p<0.05$.

$\mathrm{b}_{p}<0.01$.

${ }^{c} p<0.001$

women across all insurance categories and quartiles of median income experience increased odds of having a cesarean delivery as compared with white women.

To our knowledge, assessing effect modification by either primary insurance payer or median income quartile in the relationship between maternal race/ethnicity and outcomes has hereto not been performed, allowing for an important contribution to the literature. Moreover, the large, longitudinal sample in our analysis offers a generalizable insight into the effects of race/ethnicity on maternal outcomes. Perhaps most importantly, our multivariate analyses adjust for present-onadmission maternal-specific comorbidities from the Bateman index; adjustment by this index has not been done previously in a multistate analysis of data from the SID. Even when these comorbidities are taken into account, black women still experience poorer outcomes compared with white women.

\section{In-Hospital Maternal Mortality/Length of Stay}

Our results confirm the findings of a multitude of studies showing racial and ethnic differences in rates of maternal mortality, with black women being disproportionately affected. ${ }^{1-8,10-15}$ In our adjusted models, black women were $90 \%$ more likely than white women to die in-hospital; the same was true in stratified analyses by Medicaid and private insurance populations, with an especially strong increased likelihood of inpatient mortality in a population of black patients with private insurance. Black women were also more likely to have an expected slight increase in LOS as compared with white women, even when controlling for delivery type in adjusted analyses. This corroborated the findings of Tanner et al, who concluded that black women were more likely than white women to have LOS longer than 3 days after a scheduled cesarean delivery. ${ }^{48}$

Stratified analyses by payer type and median income quartile are especially advantageous in examining the relationship between race/ethnicity and LOS, noting the complexities of the contributing factors to LOS, ranging from patient, provider, and insurance payer preferences. ${ }^{49}$

\section{Cesarean Delivery}

Consistent with the existing literature, we found differences in racial and ethnic groups in delivery type. There were small but significant differences in the prevalence of cesarean deliveries across all racial and ethnic groups, with black women having the highest percentage (38.3\%, - Table 1). In an adjusted logistic regression model, black women were significantly more likely than white women to undergo a cesarean delivery when controlling for patient-level comorbidities and hospital characteristics; these estimates were more exaggerated in a population of Hispanic women ( - Table 4). For black women, these results were maintained in stratified analyses by categories of insurance payer and median income quartile. Among Hispanic women, results of a higher likelihood of cesarean delivery as compared with white women were consistent across all stratified models, save for in a population of Medicare patients. These estimates of the effects of race/ethnicity on the likelihood of cesarean 
delivery in both models with the full sample and stratified by median income quartile and insurance payer point to the importance of race/ethnicity as a predictor of delivery type.

Explanations about rates of cesarean delivery across racial and ethnic groups vary: studies have suggested that physician preference, maternal request, and clinical indications (such as nonreassuring fetal heart tracings or fetal distress) all play a role in determining the likelihood of a cesarean delivery. ${ }^{23,50,51}$ Roth and Henley noted the advantage of white women and more highly educated women, both of whom have better access to care and better communication with health care providers; these populations are less likely to have cesarean deliveries. ${ }^{23}$ An additional explanation for some of the racial/ ethnic disparities in rates of cesarean deliveries is based on medical need: black women are more likely to have an "unnecessary" primary cesarean delivery-when such a procedure is not medically indicated, but is preferred for financial, medico-legal, or patient demographic factors, among others. ${ }^{22}$

The epidemiological evidence of racial and ethnic disparities is manifold.

When adjusting for patient characteristics, Chung et al concluded that black women were $75 \%$ more likely than white women to have a cesarean delivery. ${ }^{20}$ In models further adding fixed effects of hospital along with random (clustering) effects of physicians, black women were $54 \%$ more likely than white women to undergo a cesarean delivery. Braveman et al found that black women were $24 \%$ more likely than white women to undergo cesarean deliveries after adjustment for sociodemographic categories and medical risk factors, as well as hospital characteristics. ${ }^{18}$ Roth and Henley added black women were $33 \%$ more likely than white women to have a primary cesarean delivery, even when adjusting for demographic and pregnancy characteristics, maternal health risks, and complications of labor and delivery. ${ }^{23}$ Finally, in adjusted models, Aron et al found that nonwhites had higher rates of cesarean delivery (OR: 1.34; 95\% CI: 1.14-1.57) than whites. ${ }^{16}$ Despite these multiple studies, contrary findings do exist. In a nationally representative sample, Witt et al discovered that there was no effect of race on the likelihood of both medically indicated and nonmedically indicated cesarean deliveries; indeed, the strongest predictor was having a previous cesarean delivery. ${ }^{17}$ At the provider level, Metz et al found no racial and ethnic differences in cesarean deliveries between providers who had low, medium, and high rates of primary cesarean deliveries. $^{52}$ These findings suggest that clinical necessity and provider preference may be most influential in determining delivery type, though mediated by sociodemographic factors.

\section{Limitations}

The SID is an administrative database comprised of data from billing records, which subjects it to concerns of validity. Data may be inaccurately coded or incomplete. Specific to variables in this study, research to validate coding practices in administrative databases has shown that delivery type has high sensitivities and positive predictive values, though select comorbidities do not. ${ }^{53,54}$ Importantly, the SID does not contain physician-level data to show provider preference for delivery type and whether this preference was clinically motivated (e.g., due to vertex presentation); the individual physician's preference has been shown to be an important factor in the likelihood of a cesarean delivery, net of hospital factors or patient comorbidities. ${ }^{52}$ Nor does the SID contain data on patient preference, which could also affect the likelihood of a cesarean delivery. ${ }^{18}$

The SID lacks information on the severity of comorbidities, as well as any data on medications or other markers of prenatal care. Neither does the SID contain behavioral or social measures on part of the patient or provider. These measures are potentially relevant factors in explaining the impact of race/ ethnicity on our outcomes. ${ }^{55,56}$ In addition, body mass index, which has been shown to be a significant predictor of adverse perinatal outcomes, ${ }^{57-61}$ is not available in the SID.

The unit of analysis in the SID is the inpatient discharge, allowing for multiple records per patient in the database with multiple pregnancies in the included states and years.

The obstetric comorbidity index used in our multivariate analyses was designed to predict maternal death or endorgan injury, ${ }^{45}$ although we have included it in multivariate analyses where death or end-organ injury is not the outcome. Even though this index has been validated, ${ }^{46}$ we cannot be certain that it captures the totality of patient conditions that might contribute to each of our outcomes.

In addition, $2.8 \%$ of our sample $(190,501)$ had missing data on race/ethnicity. Because the percentage of the total sample with missing data on race/ethnicity was below an arbitrary cutoff point of $5 \%$, we elected not to conduct a sensitivity analysis, despite our assumption that the data on race and ethnicity were not missing at random. ${ }^{62}$ Finally, we were unable to conduct multilevel analyses with hospital as a clustering variable due to model nonconvergence, though we did include hospital delivery volume as a covariate in all of our multivariate models.

\section{Strengths}

In spite of these limitations, a notable strength of our study is its comprehensive sample of hospital discharges (approximating a census) over an 8-year period in five geographically diverse states in the United States. The inclusion of patient and hospital characteristics as explanatory variables controlled for the effects of preexisting conditions and for variation in hospital delivery practice. ${ }^{63,64}$ We also controlled for year of admission to account for changes in laws that have affected insurance payer reimbursements and coverage, such as the introduction of the Patient Protection and Affordable Care Act. ${ }^{65}$

\section{Conclusion}

This study shows that racial and ethnic disparities in inpatient maternal mortality, delivery type, and LOS exist, even within strata of insurance payer and income in models controlling for confounding patient comorbidities and hospital characteristics. Specifically, racial and ethnic group differences in delivery type exist, with black women more likely than white women to die in-hospital regardless of primary insurance payer or 
residence associated with a particular income bracket. At all levels of health care, providers should be mindful of the effects of race and ethnicity on maternal outcomes, irrespective of primary insurance payer or residence in socioeconomic category. Research into the causal mechanisms of these disparities is needed with the collection of behavioral and sociological variables in hospital administrative databases.

\section{Conflict of Interest}

None.

\section{References}

1 Bryant AS, Worjoloh A, Caughey AB, Washington AE. Racial/ethnic disparities in obstetric outcomes and care: prevalence and determinants. Am J Obstet Gynecol 2010;202(04):335-343

2 Harper M, Dugan E, Espeland M, Martinez-Borges A, Mcquellon C. Why African-American women are at greater risk for pregnancyrelated death. Ann Epidemiol 2007;17(03):180-185

3 Somer SJH, Sinkey RG, Bryant AS. Epidemiology of Racial/Ethnic Disparities in Severe Maternal Morbidity and Mortality. Paper presented at Seminars in perinatology; 2017

4 Cabacungan ET, Ngui EM, McGinley EL. Racial/ethnic disparities in maternal morbidities: a statewide study of labor and delivery hospitalizations in Wisconsin. Matern Child Health J 2012;16 (07):1455-1467

5 Creanga AA, Bateman BT, Kuklina EV, Callaghan WM. Racial and ethnic disparities in severe maternal morbidity: a multistate analysis, 2008-2010. Am J Obstet Gynecol 2014;210(05):435.e1-435.e8

6 Shahul S, Tung A, Minhaj M, et al. Racial disparities in comorbidities, complications, and maternal and fetal outcomes in women with preeclampsia/eclampsia. Hypertens Pregnancy 2015;34(04):506-515

7 Shen JJ, Tymkow C, MacMullen N. Disparities in maternal outcomes among four ethnic populations. Ethn Dis 2005;15(03):492-497

8 Goffman D, Madden RC, Harrison EA, Merkatz IR, Chazotte C. Predictors of maternal mortality and near-miss maternal morbidity. J Perinatol 2007;27(10):597-601

9 Fang J, Madhavan S, Alderman MH. Maternal mortality in New York City: excess mortality of black women. J Urban Health 2000; 77(04):735-744

10 LoJO, Mission JF, Caughey AB. Hypertensive disease of pregnancy and maternal mortality. Curr Opin Obstet Gynecol 2013;25(02):124-132

11 Creanga AA, Berg CJ, Syverson C, Seed K, Bruce FC, Callaghan WM. Race, ethnicity, and nativity differentials in pregnancy-related mortality in the United States: 1993-2006. Obstet Gynecol 2012; 120(2 Pt 1):261-268

12 Berg CJ, Callaghan WM, Syverson C, Henderson Z. Pregnancyrelated mortality in the United States, 1998 to 2005. Obstet Gynecol 2010;116(06):1302-1309

13 Creanga AA, Berg CJ, Ko JY, et al. Maternal mortality and morbidity in the United States: where are we now? J Womens Health (Larchmt) 2014;23(01):3-9

14 Lang CT, King JC. Maternal mortality in the United States. Best Pract Res Clin Obstet Gynaecol 2008;22(03):517-531

15 Tucker MJ, Berg CJ, Callaghan WM, Hsia J. The Black-White disparity in pregnancy-related mortality from 5 conditions: differences in prevalence and case-fatality rates. Am J Public Health 2007;97(02): 247-251

16 Aron DC, Gordon HS, DiGiuseppe DL, Harper DL, Rosenthal GE. Variations in risk-adjusted cesarean delivery rates according to race and health insurance. Med Care 2000;38(01):35-44

17 Witt WP, Wisk LE, Cheng ER, et al. Determinants of cesarean delivery in the US: a lifecourse approach. Matern Child Health J 2015;19(01):84-93
18 Braveman P, Egerter S, Edmonston F, Verdon M. Racial/ethnic differences in the likelihood of cesarean delivery, California. Am J Public Health 1995;85(05):625-630

19 Zhang J, Troendle J, Reddy UM, et al; Consortium on Safe Labor. Contemporary cesarean delivery practice in the United States. Am J Obstet Gynecol 2010;203(04):326.e1-326.e10

20 Chung JH, Garite TJ, Kirk AM, Hollard AL, Wing DA, Lagrew DC. Intrinsic racial differences in the risk of cesarean delivery are not explained by differences in caregivers or hospital site of delivery. Am J Obstet Gynecol 2006;194(05):1323-1328

21 Coffey RM, Andrews RM, Moy E. Racial, ethnic, and socioeconomic disparities in estimates of AHRQ patient safety indicators. Med Care 2005;43(3, Suppl):I48-I57

22 Kabir AA, Pridjian G, Steinmann WC, Herrera EA, Khan MM. Racial differences in cesareans: an analysis of U.S. 2001 National Inpatient Sample Data. Obstet Gynecol 2005;105(04):710-718

23 Roth LM, Henley MM. Unequal motherhood: racial-ethnic and socioeconomic disparities in cesarean sections in the United States. Soc Probl 2012;59(02):207-227

24 Getahun D, Strickland D, Lawrence JM, Fassett MJ, Koebnick C, Jacobsen SJ. Racial and ethnic disparities in the trends in primary cesarean delivery based on indications. Am J Obstet Gynecol 2009;201(04):422.e1-422.e7

25 Huesch M, Doctor JN. Factors associated with increased cesarean risk among African American women: evidence from California, 2010. Am J Public Health 2015;105(05):956-962

26 American College of Obstetricians and Gynecologists. ACOG Committee Opinion No. 394, December 2007. Cesarean delivery on maternal request. Obstet Gynecol 2007;110(06):1501

27 Liu S, Liston RM, Joseph KS, Heaman M, Sauve R, Kramer MS; Maternal Health Study Group of the Canadian Perinatal Surveillance System. Maternal mortality and severe morbidity associated with low-risk planned cesarean delivery versus planned vaginal delivery at term. CMAJ 2007;176(04):455-460

28 Aseltine RH Jr, Yan J, Fleischman S, Katz M, DeFrancesco M. Racial and ethnic disparities in hospital readmissions after delivery. Obstet Gynecol 2015;126(05):1040-1047

29 ACOG Committee Opinion No. 649: racial and ethnic disparities in obstetrics and gynecology. Obstet Gynecol 2015;126(06): e130-e134

30 Harrell CJP, Burford TI, Cage BN, et al. Multiple pathways linking racism to health outcomes. Du Bois Rev 2011;8(01):143-157

31 Gee GC, Walsemann KM, Brondolo E. A life course perspective on how racism may be related to health inequities. Am J Public Health 2012;102(05):967-974

32 Giscombé CL, Lobel M. Explaining disproportionately high rates of adverse birth outcomes among African Americans: the impact of stress, racism, and related factors in pregnancy. Psychol Bull 2005;131(05):662-683

33 Hardeman RR, Medina EM, Kozhimannil KB. Structural racism and supporting black lives - the role of health professionals. N Engl J Med 2016;375(22):2113-2115

34 Bailey ZD, Krieger N, Agénor M, Graves J, Linos N, Bassett MT. Structural racism and health inequities in the USA: evidence and interventions. Lancet 2017;389(10077):1453-1463

35 Krieger N. Discrimination and health inequities. Int J Health Serv 2014;44(04):643-710

36 Link BG, Phelan J. Social conditions as fundamental causes of disease. J Health Soc Behav 1995(Spec No):80-94

37 Grimm K, Moore L, Scanlon K. Access to health food retailersUnites States, 2011. CDC Health Disparities and Inequalities Report-United States, 2013. Morbidity Mortality Weekly Report 2013;62(03):20-26

38 Barnett JC, Berchick ER. Health Insurance Coverage in the United States: 2016. Current Population Reports 2017. Available at: https:// www.census.gov/content/dam/Census/library/publications/2017/ demo/p60-260.pdf. Accessed March 14, 2018 
39 Fiscella K, Franks P, Gold MR, Clancy CM. Inequality in quality: addressing socioeconomic, racial, and ethnic disparities in health care. JAMA 2000;283(19):2579-2584

40 Crear-Perry J Systems and Policies Driving Black Maternal Health. Oral presentation at: 2018 New York Maternal Mortality Summit; February, 2018; New York, NY

41 Smedley BD. The lived experience of race and its health consequences. Am J Public Health 2012;102(05):933-935

42 Pickett KE, Collins JW Jr, Masi CM, Wilkinson RG. The effects of racial density and income incongruity on pregnancy outcomes. Soc Sci Med 2005;60(10):2229-2238

43 Williams DR. Race, socioeconomic status, and health. The added effects of racism and discrimination. Ann N Y Acad Sci 1999;896 (01):173-188

44 HCUP State Inpatient Databases (SID). Healthcare Cost and Utilization Project (HCUP): 2007-2014. Rockville, MD: Agency for Healthcare Research and Quality. Available at: https://www. hcup-us.ahrq.gov/sidoverview.jsp

45 Bateman BT, Mhyre JM, Hernandez-Diaz S, et al. Development of a comorbidity index for use in obstetric patients. Obstet Gynecol 2013;122(05):957-965

46 Metcalfe A, Lix LM, Johnson JA, et al. Validation of an obstetric comorbidity index in an external population. BJOG 2015;122(13): 1748-1755

47 Cheesman K, Brady JE, Flood P, Li G. Epidemiology of anesthesiarelated complications in labor and delivery, New York State, 20022005. Anesth Analg 2009;109(04):1174-1181

48 Tanner LD, Chen H-Y, Chauhan SP, Sibai BM. 944: Racial disparity in length of stay after scheduled cesarean delivery. Am J Obstet Gynecol 2018;218(01):S559-S560

49 Weiss M, Ryan P, Lokken L, Nelson M. Length of stay after vaginal birth: sociodemographic and readiness-for-discharge factors. Birth 2004;31(02):93-101

50 Barber EL, Lundsberg LS, Belanger K, Pettker CM, Funai EF, Illuzzi $\mathrm{JL}$. Indications contributing to the increasing cesarean delivery rate. Obstet Gynecol 2011;118(01):29-38

51 Boyle A, Reddy UM. Epidemiology of cesarean delivery: the scope of the problem. Semin Perinatol 2012;36(05):308-314

52 Metz TD, Allshouse AA, Gilbert SAB, Doyle R, Tong A, Carey JC. Variation in primary cesarean delivery rates by individual physician within a single-hospital laborist model. Am J Obstet Gynecol 2016;214(04):531.e1-531.e6
53 Yasmeen S, Romano PS, Schembri ME, Keyzer JM, Gilbert WM. Accuracy of obstetric diagnoses and procedures in hospital discharge data. Am J Obstet Gynecol 2006;194(04):992-1001

54 Goff SL, Pekow PS, Markenson G, Knee A, Chasan-Taber L, Lindenauer PK. Validity of using ICD-9-CM codes to identify selected categories of obstetric complications, procedures and co-morbidities. Paediatr Perinat Epidemiol 2012;26(05):421-429

55 Williams DR, Mohammed SA. Discrimination and racial disparities in health: evidence and needed research. J Behav Med 2009; 32(01):20-47

56 Bonilla-Silva E. Rethinking racism: toward a structural interpretation. Am Sociol Rev 1997;62(03):465-480

57 Vasudevan C, Renfrew M, McGuire W. Fetal and perinatal consequences of maternal obesity. Arch Dis Child Fetal Neonatal Ed 2011;96(05):F378-F382

58 Sheiner E, Levy A, Menes TS, Silverberg D, Katz M, Mazor M. Maternal obesity as an independent risk factor for caesarean delivery. Paediatr Perinat Epidemiol 2004;18(03):196-201

59 Denison FC, Norwood P, Bhattacharya S, et al. Association between maternal body mass index during pregnancy, shortterm morbidity, and increased health service costs: a populationbased study. BJOG 2014;121(01):72-81

60 Doherty DA, Magann EF, Francis J, Morrison JC, Newnham JP. Prepregnancy body mass index and pregnancy outcomes. Int J Gynaecol Obstet 2006;95(03):242-247

61 Bhattacharya S, Campbell DM, Liston WA, Bhattacharya S. Effect of Body Mass Index on pregnancy outcomes in nulliparous women delivering singleton babies. BMC Public Health 2007; 7(01):168

62 Allison PD. Missing Data: Sage University Papers Series on Quantitative Applications in the Social Sciences (07-136). Thousand Oaks, CA; 2001

63 Kozhimannil KB, Law MR, Virnig BA. Cesarean delivery rates vary tenfold among US hospitals; reducing variation may address quality and cost issues. Health Aff (Millwood) 2013;32(03): 527-535

64 Little SE, Orav EJ, Robinson JN, Caughey AB, Jha AK. The relationship between variations in cesarean delivery and regional health care use in the United States. Am J Obstet Gynecol 2016;214(06): 735.e1-735.e8

65 2010. March 23, The Patient Protection and Affordable Care Act (PPACA), Pub. L. No. 111-148, 124 Stat. 119 